\title{
Investigation into the Lubrication Regime for Lumbar Total Disc Replacement of the Spine
}

\author{
Markus Kraft \\ ${ }^{I}$ (Innovation, Materials \& Surfaces, DePuy Synthes, Synthes GmbH, Luzernstrasse 21, CH-4528 Zuchwil, \\ Switzerland)
}

\begin{abstract}
The lubrication regime of articulating surfaces plays an important role for the understanding of the tribological conditions and the resulting wear performance of total disc replacement (TDR) of the spine.

In the present study the lubrication regime for lumbar TDRs with ball and socket design have been assessed by applying the Hamrock-Dawson elasto-hydrodynamic lubrication theory. The effect of varying articulation radii and different material combinations on the lubrication regime have been investigated considering load and motion cycles for a representative in vivo situation as well as for in vitro wear testing situations.

Independent of geometry or bearing material combinations, the formation of a stable lubrication film is very unlikely for lumbar TDR. The study also reveals that in vitro testing conditions must be carefully selected in order to correlate with the lubrication regime for corresponding in vivo situations.
\end{abstract}

Keywords: ball-in-socket joint, elasto-hydrodynamic lubrication, load and motion cycle, lumbar total disc replacement, spine, biotribology.

\section{INTRODUCTION}

Spinal fusion is the gold standard for treating degenerative conditions of the aging spine by encouraging adjacent vertebra to grow together. Since the development of the SB Charité I artificial disc in the early 1980ies [1], total disc replacement (TDR) has evolved as a promising alternative to spinal fusion. Its aim is to preserve spinal motion at the treated levels, and possibly to reduce adjacent-level degeneration $[2,3]$.

During the last 15 years a variety of lumbar TDR designs have been developed and introduced for clinical use [4]. The majority of the current approaches to arthroplasty in the lumbar spine use principles similar to those used in hip arthroplasty with spherical shaped articulating surfaces. The material combinations used include UHMWPE, CoCrMo alloys, and high performance ceramics to mention the most common.

TDRs must resist harsh operating conditions over their entire service life: The corrosive environment, high mechanical stress, and cyclic loading must be considered when developing and validating new implant designs. Besides the geometrical factors and the material selection for articulating implant surfaces, the type of lubrication regime is an important element for the understanding of the tribological conditions and the resulting wear mechanisms for artificial joints.

Analogous to published work for artificial hips [5], or wrist implants [6] the lubrication regimes for lumbar TDRs with ball and socket design have been estimated in previous studies [7, 8]. In [9] the lubrication regime for cervical TDR was analyzed. All these studies applied the Hamrock-Dawson elasto-hydrodynamic lubrication theory in order to calculate the lubrication film thickness for different material combinations. However, these studies are of limited practical relevance as they do not consider the kinematics and the load conditions for typical daily activities in an in vivo situation.

The aim of this study is to close this gap by considering the kinematics and loads in the lumbar spine for a typical daily activity. Based on this information the likely lubrication regimes for lumbar TDRs with different bearing material combinations are calculated. In addition these lubrication regimes are compared with the expected lubrication regimes during in vitro wear testing of lumbar TDRs as proposed by the ISO 18192 standard. This is of high importance as in vitro testing shall mimic the tribological conditions of an in vivo situation.

\subsection{Lubrication model}

\section{METHODS}

For the calculation of the lubrication regime a lumbar TDR with a ball and socket design was considered. For the calculation of the minimum lubrication thickness $h_{\min }$ the Hamrock-Dawson elastohydrodynamic lubrication theory was applied, assuming circular contacts between the articulating surfaces (1). For further details refer to e.g. [7] or [10]. 


$$
\frac{h_{\min }}{r^{\prime}}=2.80\left(\frac{\eta u}{E^{\prime} r^{\prime}}\right)^{0.65}\left(\frac{F}{E^{\prime} r^{\prime 2}}\right)^{-0.21}
$$

In (1) $\mathrm{F}$ denotes the axial force and $\eta$ the lubricant's viscosity. As discussed in [7] the lubricant in a TDR is assumed to be interstitial fluid with a viscosity $\eta$ of $1.24 \mathrm{mPas}$ which is approximately 8 times less viscous compared to the synovial fluid of a diarthrosis. In the case of in vitro wear tests of TDRs the viscosity of the test medium containing $20 \%$ bovine serum is $1.0 \mathrm{mPas}$ [11]. Fortunately this is very close to the viscosity assumed for interstitial fluid for the in vivo case. Please note that in both cases (in vivo \& in vitro) the fluid was assumed to be Newtonian and possible shear thinning effects were neglected.

According to [7] the entraining velocity $\mathrm{u}$ in equation (1) is calculated according to (2), where $\omega$ stands for the angular velocity and $\mathrm{r}_{1}$.denotes the radius of the ball.

$u=\frac{\omega r_{1}}{2}$

$E^{\prime}$ in (1) denotes the equivalent elastic modulus which is determined according to (3), with the Young's moduli $\mathrm{E}_{1}$ (ball material) and $\mathrm{E}_{2}$ (socket material) and the respective Poisson's ratios $v_{1}$ and $v_{2}$. The equivalent radius $\mathrm{r}^{\prime}$ is calculated using (4) where $\mathrm{r}_{1}$ is the ball radius, $\mathrm{r}_{2}$ the socket radius, and $\mathrm{c}_{\text {radial }}$ the radial clearance $\left(c_{\text {radial }}=\mathrm{r}_{1}-\mathrm{r}_{2}\right)$.

$$
\begin{aligned}
& E^{\prime}=\frac{2 E_{1} E_{2}}{E_{2}\left(1-v_{1}^{2}\right)+E_{1}\left(1-v_{2}^{2}\right)} \\
& r^{\prime}=\frac{r_{1} r_{2}}{r_{2}-r_{1}}=\frac{r_{1}}{c_{\text {radial }}}\left(r_{1}+c_{\text {radial }}\right)
\end{aligned}
$$

By comparing the minimum film thickness $\mathrm{h}_{\min }$ with the compound surface roughness $\mathrm{R}_{\mathrm{a}, \mathrm{c}}$ the resulting $\lambda$-values were obtained using (5) where $\mathrm{R}_{\mathrm{a}, 1}$ and $\mathrm{R}_{\mathrm{a}, 2}$ denote the surface roughness of the articulating surfaces.

$$
\lambda=\frac{h_{\min }}{R_{a, c}} \text { with } R_{a, c}=\sqrt{R_{a, 1}^{2}+R_{a, 2}^{2}}
$$

Table 1: Parameters for different bearing materials

\begin{tabular}{cccc}
\hline Material & E-modulus & Poisson's ratio & Surface roughness \\
\hline CoCrMo & $241 \mathrm{GPa}$ & 0.3 & $0.01 \mu \mathrm{m}$ \\
UHMWPE & $0.58 \mathrm{GPa}$ & 0.46 & $0.75 \mu \mathrm{m}$ \\
Ceramic $^{1}$ & $358 \mathrm{GPa}$ & 0.22 & $0.005 \mu \mathrm{m}$ \\
PEEK & $3.5 \mathrm{GPa}$ & 0.4 & $0.25 \mu \mathrm{m}$ \\
\hline
\end{tabular}

${ }^{I}$ Biolox Delta®, Ceramtec, Germany

Table 2: Radial clearance for different bearing material combinations

\begin{tabular}{ccc}
\hline Material combination & Radial clearance $\mathbf{c}_{\text {radial }}$ & Reference \\
\hline UHMWPE-on-CoCrMo & $100 \mu \mathrm{m}$ & {$[7,13,14]$} \\
CoCrMo-on-CoCrMo & $30 \mu \mathrm{m}$ & {$[13,15]$} \\
Ceramic-on-Ceramic ${ }^{1}$ & $30 \mu \mathrm{m}$ & {$[13]$} \\
PEEK-on-PEEK & $100 \mu \mathrm{m}$ & {$[16]$} \\
\hline${ }^{1}$ Biolox Delta®, Ceramtec, Germany & &
\end{tabular}


The obtained $\lambda$-values allowed for prediction of the likely lubrication regimes for different configurations as expressed in the Stribeck-curve [12]. Accordingly for $\lambda>3$ a hydrodynamic lubrication regime is expected. $\lambda<1$ stands for boundary lubrication, and $\lambda$ between 1 and 3 signifies mixed lubrication. From (1), (4), \& (5) it follows that for a given bearing material combination fluid-film lubrication is encouraged by making the ball as large as practicably possible, the radial clearance as small as possible, and the bearing surface as smooth as possible.

\subsection{Characteristics of bearing materials}

For this study several bearing material combinations were considered. The selection was based on two factors:

(a) material combinations which are in clinical use for TDR (UHMWPE-on-CoCrMo, CoCrMo-on-CoCrMo)

(b) material combinations which have an investigational status for TDR (PEEK-on-PEEK, Ceramic-onCeramic).

The various input parameters needed for the lubrication model like E-modulus, Poisson's ratio, and typical surface roughness for the different materials are summarized in Table 1. Similar to THR, clearance values for TDR vary for different bearing material combinations. Typical values for the radial clearance $c_{\text {radial }}$ are listed in Table 2.

\subsection{Load and motion cycle (in vivo)}

\subsubsection{Range of motion (ROM)}

While for hip joints there is consent on the ROM and loads during the gait cycle (ISO 14242-1 2012), a representative load and motion cycle for the lumbar spine is not readily available. In order to define such a load and motion cycle the scientific literature on kinematics and loading of the lumbar spine was consulted.

For the kinematics the full active ROM of the lumbar spine and the ROM of the individual lumbar segments was assessed by referring to various studies published in literature (e.g. [17]-[21]). In these studies the obtained values for the full active lumbar ROM ranged from $42^{\circ}$ to $67^{\circ}$ for flexion (forward bending of the spine) and from $12^{\circ}$ to $29^{\circ}$ for extension (bending the spine backwards). The ROM values depended on gender [18], age [18,20], and lumbar level [17,20]. A typical value for the full active ROM of a single lumbar segmentl was $12^{\circ}$ in flexion and $5^{\circ}$ in extension [17,20]. According to the findings in [21] the full active ROM was found to be much larger than the ROM for typical daily activities and even larger than activities with significant motion of the spine like e.g. changing from standing to sitting, squatting or picking up an object. Following the investigation in [21] and considering the data for bending forward (flexion maneuver), the ROM for a single lumbar segment representing a motion profile for a typical activity like picking up an object from the floor was set to $0^{\circ}$ at the start of the flexion maneuver and to $11^{\circ}$ for the flexed posture.

Table 3: Summary of in vivo intradiscal pressure measurements for selected postures, number in brackets represent standard deviations.

\begin{tabular}{ccccc}
\hline Reference: & Nachemson [22] & Wilke et al [23] & Sato et al [24] & Polga et al [25] \\
\hline Laying (prone/supine) & $250 \mathrm{~N}$ & $0.11 \mathrm{MPa}$ & $0.09(0.03) \mathrm{MPa}$ & $0.2(0.03) \mathrm{MPa}$ \\
Standing upright & $500 \mathrm{~N}$ & $0.5 \mathrm{MPa}$ & $0.53(0.18) \mathrm{MPa}$ & $0.86(0.06) \mathrm{MPa}$ \\
Standing flexed & $1000 \mathrm{~N}^{2}$ & $1.1 \mathrm{MPa}$ & $1.32(0.22) \mathrm{MPa}^{3}$ & $1.09(0.05) \mathrm{MPa}^{4}$ \\
\hline Mean body weight & $70 \mathrm{~kg}$ & 70 & 73 & 73 \\
No. of participants & 1 & 1 & 28 & 6 \\
Conversion factor & - & $897 \mathrm{~N} / \mathrm{MPa}$ & $833 \mathrm{~N} / \mathrm{MPa}$ & $530 \mathrm{~N} / \mathrm{MPa}$ \\
\hline
\end{tabular}

values for lower thoracic levels (T9 to T12); ${ }^{2}$ bent forward $40^{\circ},{ }^{3}$ angle of inclination during bending forward not indicated, ${ }^{4}$ bent forward $30^{\circ}$.

Table 4: Force values for standing upright and standing flexed, derived from of in vivo intradiscal pressure measurements.

\begin{tabular}{cccccc}
\hline Reference: & $\begin{array}{c}\text { Nachemson } \\
{[\mathbf{2 2}]}\end{array}$ & Wilke et al [23] & Sato et al [24] & $\begin{array}{c}\text { Polga et al } \\
{[25]}\end{array}$ & Mean value \\
\hline Standing upright & $500 \mathrm{~N}$ & $449 \mathrm{~N}$ & $442 \mathrm{~N}$ & $456 \mathrm{~N}$ & $446 \mathrm{~N}$ \\
Standing flexed & $1000 \mathrm{~N}$ & $987 \mathrm{~N}$ & $1100 \mathrm{~N}$ & $578 \mathrm{~N}$ & $1007 \mathrm{~N}$ \\
\hline
\end{tabular}




\subsubsection{Load cycle}

For defining the load of the lumbar spine in a significant maneuver like bending forward, the literature on in vivo intradiscal pressure measurements was consulted (see [22]-[25]). In Table 3 the measured in vivo intradiscal pressures for relevant postures (laying, standing, standing flexed) are listed. In addition for each study the number of participants and the mean body weight are summarized. In a first approximation the difference in pressure between standing relaxed and laying must be due to the gravity of the upper trunk including arms and head. For a person of 70 to $73 \mathrm{~kg}$ the acting gravity was estimated to $350 \mathrm{~N}$. Considering the pressure difference between laying and standing relaxed and using the value for the acting gravity, the factor to convert pressure into force was estimated for each study (see Table 3). These conversion factors were used to transform the measured pressure values to force values. The force values of each study were weighted with the participants number in order to calculate a mean value for the force for standing upright and for standing flexed postures (Table 4).

Information on load levels for different postures is not sufficient. More importantly the time dependent progression of the loads and the resulting angular velocity during the flexion maneuver must be considered. In [26] Rohlmann et al. have reported measurements of in vivo loads with a telemeterized vertebral body replacement (VBR) during a flexion maneuver (refer to Fig. 1, measured data). As the affected spinal segments were additionally stabilized with a posterior pedicle screw based rod system, the measured magnitude of loads from [26] were not considered representative for our purpose. This is because only a part of the load was born by the VBR device. However the progression of loads with time and the relative change of the loads during the flexion maneuver were considered to be relevant. Based on the information on the loads from in vivo intradiscal pressure measurements from Table 4, the load profile from [26] was corrected by a linear factor as shown in Fig. 1.

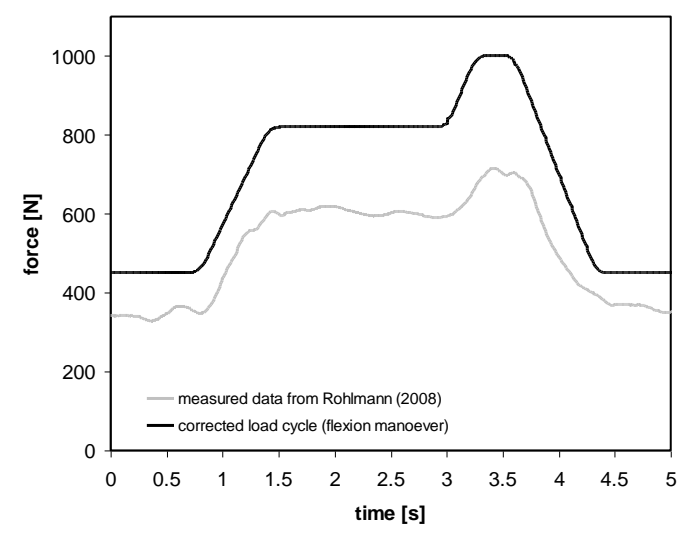

Figure 1: In vivo load cycle for flexion manoever according to Rohlmann [26] and corrected load profile to mean load values from Table 4.

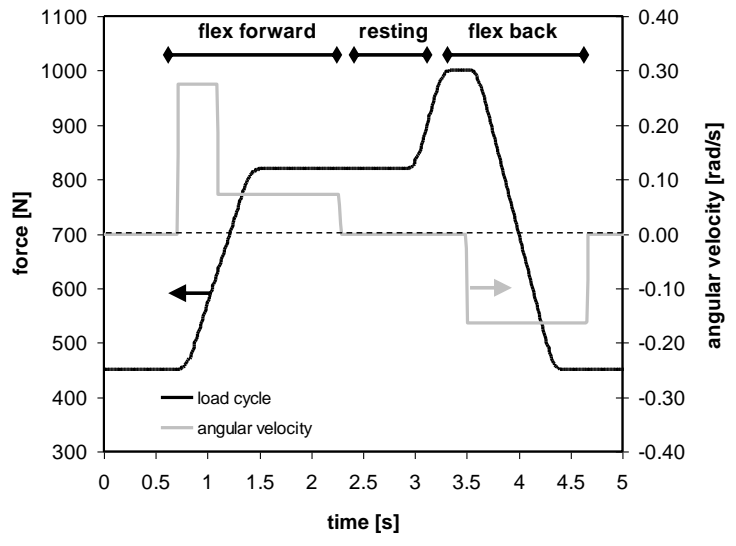

Figure 2: Proposed load and motion profile for the lumbar spine for flexing forward

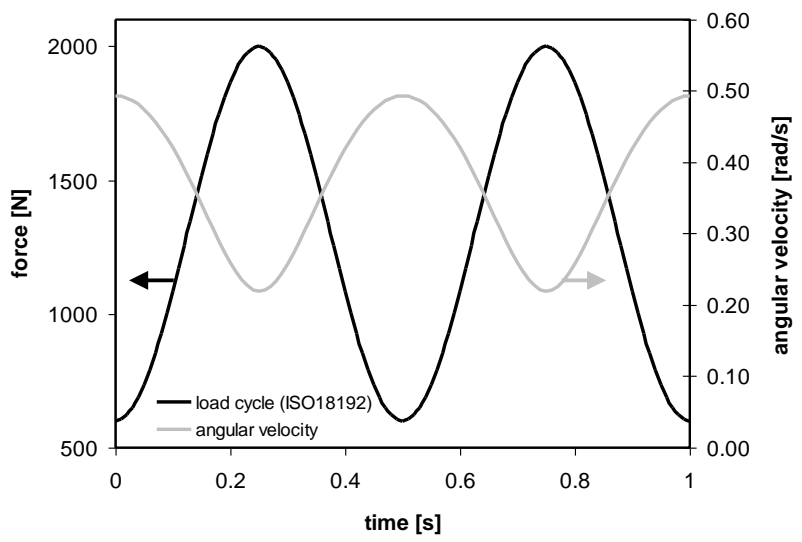

Figure 3: Load and motion profile for in vitro testing of lumbar TDRs at 1Hz, following the ISO 18192 standard. 


\subsubsection{Angular velocity}

In the experiments performed by Rohlmann et al. [26] the test person stood relaxed for approximately $0.7 \mathrm{~s}$, then flexed their upper body forward as far as possible (up to $2.3 \mathrm{~s}$ ), stayed in this position until about $3.3 \mathrm{~s}$, flexed back (up to 4.7s), and stood relaxed again. This information was used to estimate the angular velocity of a single lumbar segment throughout the flexion maneuver by considering a maximum segmental flexion angle of $11^{\circ}$ as described above. The data for the loads and for the angular velocity as a function of time over the entire flexion maneuver are illustrated in Figure 2.

\subsection{Load and motion cycle (in vitro wear testing)}

In vitro wear tests should mimic the wear conditions of TDRs in an in vivo situation. It is therefore relevant that the lubrication regimes for in vitro wear testing and for an in vivo load situation match. In vitro wear testing of lumbar TDRs is usually performed by applying one of the test protocols as described either in ISO 18192-1 (2011) or ASTM F2423 (2011) standards. In this study the lubrication regime has been assessed exemplarily for the standard load and displacement profile for lumbar total disc replacements of the ISO standard test protocol. For the calculation of the angular velocity the sliding distance per time increment was calculated for test frequencies of $1 \mathrm{~Hz}$ and $2 \mathrm{~Hz}$ following the method described by Paré [27]. The sliding velocity was calculated for various positions on the bearing ball. It was found that for the pole of the ball the sliding velocity was largest. Based on this finding the pole position of the bearing ball was taken as the reference point for subsequent assessments as it represents the position with the largest sliding velocity or entraining velocity respectively. The resulting load and angular velocity profiles throughout a wear test cycle are shown exemplarily for $1 \mathrm{~Hz}$ in Fig. 3.

\subsection{In vivo wear situation}

\section{RESULTS}

Considering equation (1) and the input parameters for different bearing materials from Tables $1 \& 2$ the minimum lubrication thickness $h_{\min }$ and the resulting $\lambda$-values were calculated over the entire load and motion cycle representing a flexion maneuver as shown in Fig. 2. In Fig. 4 the calculated $\lambda$-values for different bearing material combinations (ball radius $\mathrm{r}_{1}$ of $16.5 \mathrm{~mm}$ which is typical for lumbar TDR) are shown.

The largest $\lambda$-values are found for an all ceramic bearing followed by the CoCrMo-on-CoCrMo set-up. PEEK-on-PEEK and the classical UHMWPE-on-CoCrMo lead to drastically reduced $\lambda$-values. In all cases the calculated $\lambda$-values are considerably smaller than unity which is the limiting value below which - following the applied model - a boundary lubrication regime is predicted. In addition to the different material combinations of the bearings, the influence of different ball radii on the $\lambda$-values was assessed.

Respective results are shown in Fig. 5 exemplarily for a CoCrMo-on-CoCrMo pairing. As expected from equation (1), a reduction of the ball radius resulted in a reduced $\lambda$-value and vice versa. Notably, the effect of different bearing materials on the $\lambda$-value seemed to be much more pronounced than the influence of the ball radius.

\subsection{In vitro wear situation}

The calculated $\lambda$-values for the motion profile of the in vitro wear testing set-up are shown in Fig. 6 $(1 \mathrm{~Hz})$ and Fig. $7(2 \mathrm{~Hz})$. Again the same four bearing material pairings were considered and the ball radius was set to $16.5 \mathrm{~mm}$.

The ranking order remained the same as in the in vivo case with largest $\lambda$-values for Ceramic-onCeramic and smallest $\lambda$-values for UHMWPE-on-CoCrMo. However, in the case of $1 \mathrm{~Hz}$ test frequency the $\lambda$ values for the Ceramic-on-Ceramic bearing lay between 0.65 and 1.43 indicating a mixed lubrication regime through a considerable part of the wear cycle. At $2 \mathrm{~Hz}$ test frequency the $\lambda$-values were between 1.04 and 2.23 for the same material pairing and between 0.61 and 1.30 for $\mathrm{CoCrMo}-\mathrm{on}-\mathrm{CoCrMo}$ thus indicating mainly mixed lubrication. For UHMWPE-on-CoCrMo and PEEK-on-PEEK bearings the model predicted boundary lubrication for both test frequencies as $\lambda$-values were well below unity in both cases (see Table 5 for details). 


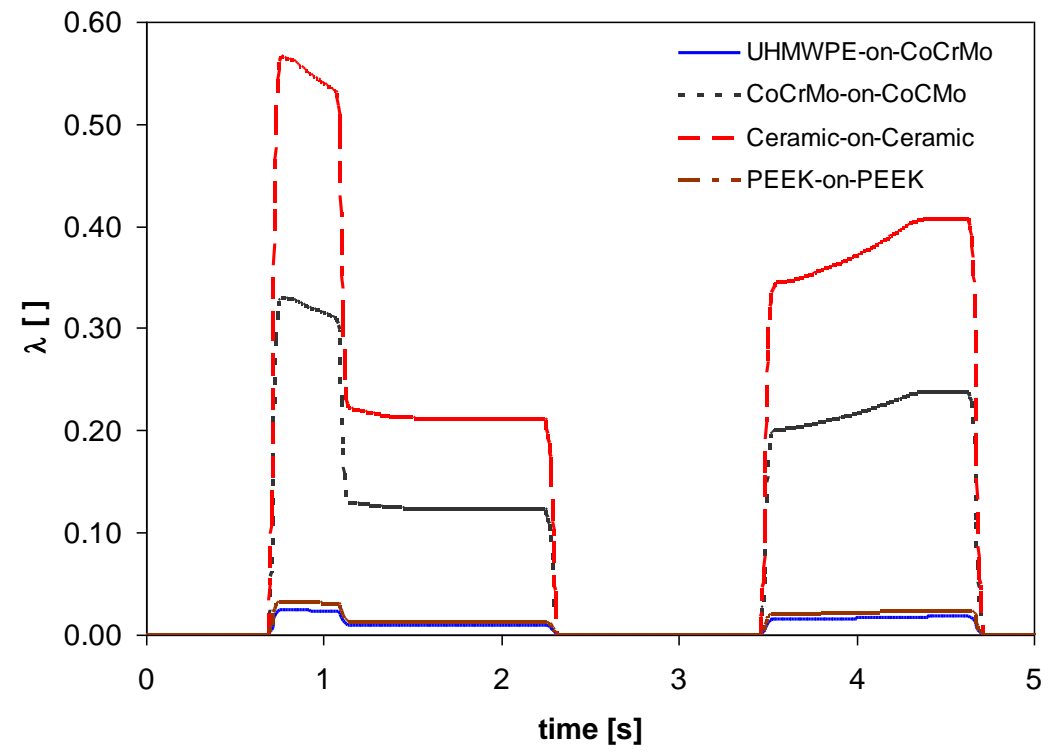

Figure 4: $\lambda$-values as calculated with the Hamrock-Dawson equation for picking up an object and considering the load and motion cycle as shown in Fig. 2, comparison of different bearing material combinations for an articulation radius of $16.5 \mathrm{~mm}$.

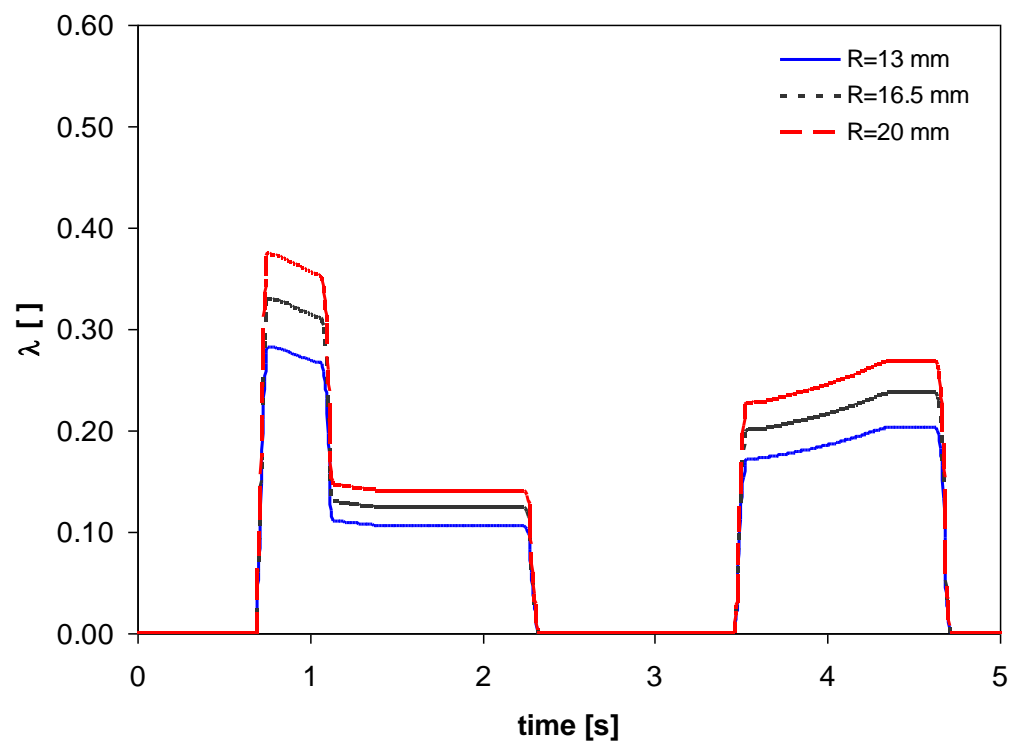

Figure 5: ג-values for picking up an object and considering the load and motion cycle as shown in Fig. 2, influence of articulation radii, CoCrMo-on-CoCrMo. 


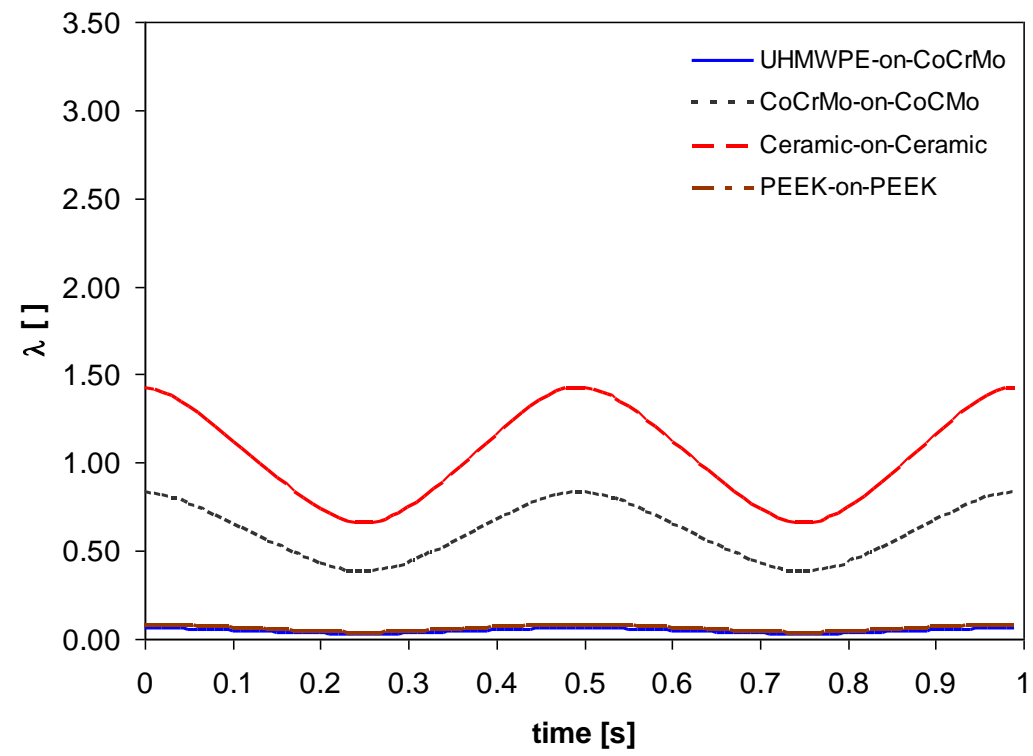

Figure 6: $\lambda$-values for in vitro testing following ISO 18192 Standard, lumbar test protocol, comparison of different bearing material combination for an articulation radius of $16.5 \mathrm{~mm}, 1 \mathrm{~Hz}$ test frequency.

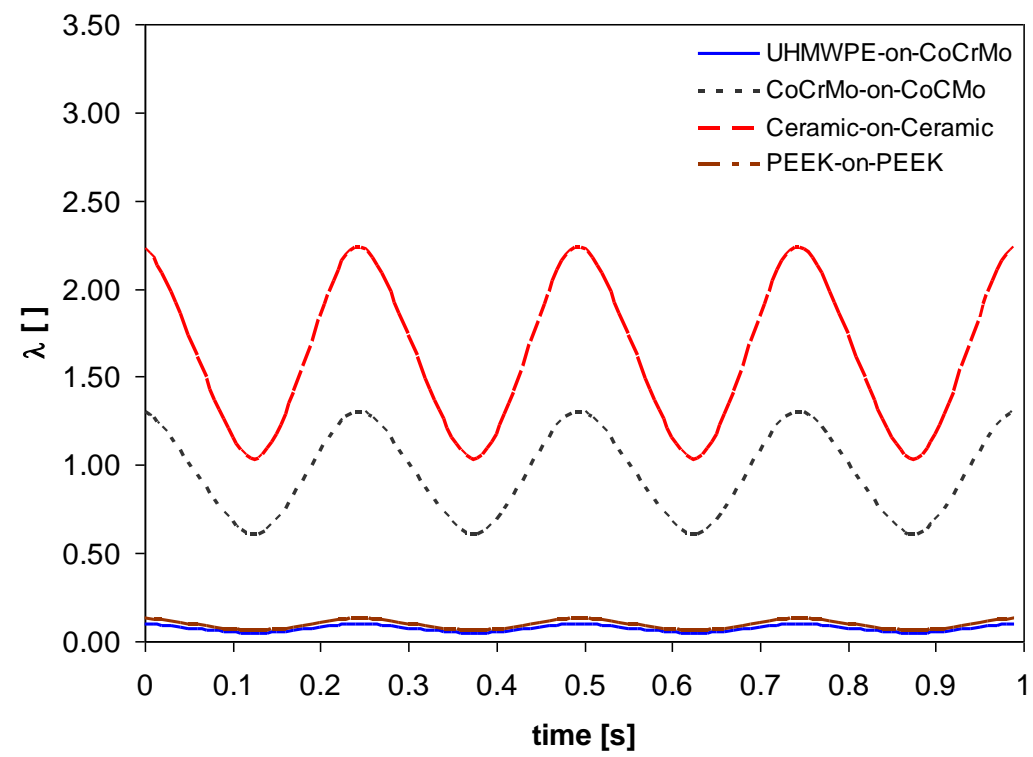

Figure 7: $\lambda$-values for in vitro testing following ISO 18192 Standard, lumbar test protocol, comparison of different bearing material combinations for an articulation radius of $16.5 \mathrm{~mm}, 2 \mathrm{~Hz}$ test frequency.

Table 5: Summary of $\lambda$-values and lubrication regimes for an articulating radius of $16.5 \mathrm{~mm}$ and different material combinations of the bearing

\begin{tabular}{ccccc}
\hline \multirow{2}{*}{ Material pairing: } & \multicolumn{2}{c}{ In vivo } & \multicolumn{2}{c}{ In vitro } \\
\cline { 2 - 5 } & $\lambda$-value range & lubrication regime & $\lambda$-value range & lubrication regime \\
\hline UHMWPE-on- & $0.21-0.56$ & boundary lubrication & $1.41-2.23$ & mixed lubrication \\
CoCrMo & & & & \\
CoCrMo-on-CoCrMo & $0.12-0.32$ & boundary lubrication & $0.61-1.30$ & $\begin{array}{c}\text { boundary/mixed } \\
\text { lubrication }\end{array}$ \\
Ceramic-on-Ceramic ${ }^{1}$ & $0.01-0.03$ & boundary lubrication & $0.06-0.13$ & boundary lubrication \\
PEEK-on-PEEK & $0.01-0.02$ & boundary lubrication & $0.05-0.10$ & boundary lubrication \\
\hline Biolox Delta $®$, Ceramtec, Germany & & &
\end{tabular}




\section{DISCUSSION}

In this paper the likely lubrication regimes for lumbar TDRs for a representative in vivo situation as well as for an in vitro wear testing situation have been investigated. While for total hip replacement a standard gait cycle has been defined, no such standard load and motion cycle was readily available for lumbar TDRs. Therefore, a representative load and motion cycle has been defined based on existing literature on kinematics and loads for the lumbar spine. For the subsequent analysis the Hamrock-Dawson equation has been considered and four different bearing material combinations as well as three different articulating radii have been assessed. For the in vivo situation bending forward (= flexion movement) was chosen as a representative model case for the following reasons: Bending forward involves a comparably high load as well as a significant ROM of the lumbar spine and represents a maneuver with significant angular velocity. In addition this maneuver is a standard situation for spinal motion for which sufficient information is available from literature.

The calculated angular velocities for this model case were a factor of 6 to 7 lower than the expected angular velocities during the gait cycle of the hip (refer e.g. to [28] \& [29]). Consequently, in the case of lumbar TDR the resulting minimum lubrication thickness $h_{\min }$ and the corresponding $\lambda$-values are comparatively small, indicating a boundary lubrication regime for all assessed bearing material combinations. This result signifies that in vivo any lubrication regime other than boundary lubrication is very unlikely for lumbar TDR. This finding is irrespective of articulating radii (investigated range between 13 and $20 \mathrm{~mm}$ ) or bearing material combinations and contrasts with the situation for total hip replacement where in specific cases mixed lubrication or fluid film lubrication are assumed [29].

In contrast to the in vivo case the assessment for the in vitro situation leads to a different result. For all material combinations and at a test frequency of $1 \mathrm{~Hz}$ the $\lambda$-values are by a factor of 2.5 larger than for the corresponding in vivo assessment. In all cases boundary lubrication is predicted by the model except for a ceramic-on-ceramic bearing for which - at least in part - mixed lubrication is predicted. If the testing frequency is doubled, a mixed lubrication is predicted in the case of a ceramic-on-ceramic bearing and a combination of boundary and mixed lubrication is found for a CoCrMo-on-CoCrMo bearing.

\section{CONCLUSION}

By applying the Hamrock-Dawson elasto-hydrodynamic lubrication theory to a lumbar TDR set-up and by considering a load and motion profile which is representative for an in vivo situation it was shown in this study that the formation of a lubrication film was very unlikely. On the contrary, the lubrication was shown to be mainly governed by boundary lubrication for which the articulating surfaces are assumed to be in close contact.

As the lubrication regime may dramatically influence the wear behavior of an artificial joint and as the in vitro wear is intended to mimic the in vivo wear situation, the lubrication regimes for both the in vivo and the in vivo situations were compared. The observed differences between the two in terms of $\lambda$-values and lubrication regimes must be carefully considered when choosing the parameters for wear testing of lumbar TDR.

\section{REFERENCES}

[1] K. Büttner-Janz, History, in K. Büttner-Janz, S.H. Hochschuler, P.C. McAfee (Eds.), The artificial disc, (Berlin: Springer-Verlag, 2003) 1-10.

[2] P. Frelinghuysen, R.C. Huang, F.P. Girardi, F.P. Cammisa, Lumbar total disc replacement part I: rationale, biomechanics, and implant types, Orthopedic Clinics of North America, 36, 2005, 293-299.

[3] P.A. Anderson, J.P. Rouleau, Intervertebral disc arthroplasty, Spine 29(23), 2006, 2279-2786.

[4] K.D. Van Den Eerenbeemt, R.W. Ostelo, B.J. Van Royen, W.C. Peul, M.W. Van Tulder, Total disc replacement surgery for symptomatic degenerative lumbar disc disease: a systematic review of the literature, European Spine Journal 19, 2010, 1262-1280.

[5] S.C. Scholes, A. Unsworth, Comparison of friction and lubrication of different hip prostheses, Proceedings of the Institution of Mechanical Engineers 214 Part H: Journal of Engineering in Medicine, 2000, 49-57.

[6] T. Pylios, D.E. Shepherd, Prediction of lubrication regimes in wrist implants with spherical bearing surfaces, Journal of Biomechanics 37(3), 2004, 405-411.

[7] A. Shaheen, D.E.T. Shepherd, Lubrication regimes in lumbar total disc arthroplasty, Proceedings of the Institution of Mechanical Engineers 221 Part H: Journal of Engineering in Medicine, 2007, 621-627.

[8] M. Bushelow, W. Nechtow, J. Walker, P8: Metal-on-Metal lumbar TDR design: a theoretical analysis of the affect of diameter clearance and surface finish on lubrication, Proceedings of the NASS 23rd Annual Meeting, The Spine Journal 8, 2008, 105S.

[9] H. Xin, D.E.T. Shepherd, K.D. Dean, PEEK (polyetheretherketone) based cervical total disc arthroplasty: Contact stress and lubrication analysis, Open Biomed. Eng. Journal 6, 2012, 73-79. 
[10] D. Dowson, Z.M. Jin, Metal-on-metal hip joint tribology, Proceedings of the Institution of Mechanical Engineers Vol. 220(2) Part H: Journal of Engineering in Medicine, 2006, 107-118.

[11] J.Q. Yao, M.P. Laurent, T.S. Johnson, C.R. Blanchard, R.D. Crowinshield, The influence of lubricant and material on polymer/CoCr sliding friction, Wear 255, 2003, 780-784.

[12] Z.M. Jin, M. Stone, E. Ingham, J. Fisher, Biotribology, Current Orthopaedics 20(1), 2006, 32-40.

[13] C. Brockett, S. Williams, Z. Jin, G. Isaac, J. Fisher, Friction of total hip replacements with different bearings and loading conditions, J Biomed Mater Res Part B: Appl Biomater 81B, 2007, 508-515.

[14] S.A. Wenzel, D.E.T. Shepherd, Contact stress in lumbar total disc arthroplasty, Bio-Med Mat Eng 17, 2007, 169-173.

[15] J.L. Lee, F. Billi, S.N. Sangiorgio, W. McGarry, D.J. Krueger, P.T. Miller, H. McKellop, E. Ebramzadeh, Wear of an experimental metal-on-metal artificial disc for the lumbar spine, Spine 33(6), 2008, 597-606.

[16] M. Kraft, D.K. Koch, M. Bushelow, An investigation into PEEK-on-PEEK as a bearing surface candidate for cervical total disc replacement, Spine J 12, 2012, 603-611.

[17] M. Pearcy, I. Portec, J. Shepherd, Three-dimensional x-ray analysis of normal movement in the lumbar spine, Spine 9(3), 1984, 294-300.

[18] P. Russell, M.J. Peardy, A. Unsworth, Measurement of the range and coupled movements observed in the lumbar spine, British Journal of Rheumatology 32, 1993, 490-497.

[19] J.K. Ng, V. Kippers, C.A. Richardson, M. Parnianpour, Range of motion and lordosis of the lumbar spine, Spine 26(1), 2001, 53-60.

[20] K.W. Wong, J.Y. Leong, M. Chan, K.D.K. Luk, W.W. Lu, The flexion-extension profile of lumbar spine in 100 healthy volunteers, Spine 29(15), 2004, 1636-1641.

[21] J.E. Bible, D. Biswas, C.P. Miller, P.G. Whang, J.N. Grauer, Normal functional range of motion of the lumbar spine during 15 activities of daily living, Journal of Spinal Disorders and Technologies 23(2), 2010, 106-112.

[22] A.L. Nachemson, Disc pressure measurements, Spine 6(1), 1981, 93-97.

[23] H.J. Wilke, P. Neef, M. Caimi, T. Hoogland, L.E. Claes, New in vivo measurements of pressures in the intervertebral disc in daily life, Spine 24(8), 1999, 755-762.

[24] K. Sato, S. Kikuchi, T. Yonezawa, In vivo intradiscal pressure measurement in healthy individuals and in patients with ongoing back problems, Spine 24(23), 1999, 2468-2474.

[25] D.J. Polga, B.P. Beaubien, P.M. Kallemeier, K.P. Schellhas, W.D. Lew, G.R. Buttermann, K.B. Wood, Measurement of in vivo intradiscal pressure in healthy thoracic intervertebral discs, Spine 29(12), 2000, 1320-1324.

[26] A. Rohlmann, F. Graichen, R. Kayser, A. Bender, G. Bergmann, Loads on a telemeterized vertebral body replacement measured in two patients, Spine 33(1), 2008, 1170-1179.

[27] P.E. Paré, F.W. Chan, S. Bhattacharya, V.K. Goel, Surface slide track mapping of implants for total disc arthroplasty, Journal of Biomechanics 42, 2009, 131-139.

[28] D. Jalali-Vahid, M. Jagatia, M.Z. Jin, D. Dawson, Prediction of lubrication film thickness in a ball-insocket model with a soft lining representing human natural and artificial hip joints, Proceedings of the Institution of Mechanical Engineers 215 Part J:Journal of Engineering Tribology, 2001, 363-372.

[29] L. Mattei, F. Di Puccio, B. Piccigallo, E. Ciulli, Lubrication and wear modelling of artificial hip joints: A review, Tribology International 44(5), 2011, 532-549. 